\title{
AN OPTIMIZED HYBRID BEAMFORMING FOR MILLIMETER WAVE MU- MASSIVE MIMO SYSTEM
}

\author{
Saeed Ali Saeed Alquhaif \\ University of Lahore, Lahore, (Pakistan) \\ E-mail: s.alquhaif@gmail.com \\ Ishtiaq Ahmad \\ University of Lahore, Lahore, (Pakistan) \\ E-mail: ishtiaq.ahmad@ee.uol.edu.pk \\ Mahnoor Rasheed \\ University of Lahore, Lahore, (Pakistan) \\ E-mail: mahnoor.rasheed@es.uol.edu.pk \\ Ali Raza \\ University of Lahore, Lahore, (Pakistan) \\ E-mail: ali.raza@ee.uol.edu.pk
}




\section{ABSTRACT}

Millimeter wave (mmWave) and massive multiple-input multiple-output communications (massive MIMO) have been adopted as the most important keys for the next generation $(5 \mathrm{G})$ wireless networks due to high spectrum availability and massive use of space diversity for high throughput communications. Massive MIMO must be implemented parallel with mmWave to overcome high path-loss issue in mmWave band by improving the antennas gain. Digital precoding in massive MIMO shows high performance at the cost of hardware complexity due to the requirement of huge number of radio frequency (RF) chains and analog-to-digital converters (ADCs). On the other hand, analog precoding has less complexity with limited performance as it supports only one data stream. In compromise between these two, a hybrid precoding for multiuser massive MIMO (MU-massive MIMO) systems with low-complexity and reasonable performance becomes necessary for next generation networks. We propose an optimized hybrid precoding and combining scheme for fully connected structure. The design of combined analog and digital precoding for the mmWave MU-massive MIMO system becomes a non-convex problem. Therefore, we proposed a suboptimal design, where we split the transmitter and receiver hybrid precoding and decoding and adopt orthogonal matching pursuit algorithm for their design. The simulation results show that the performance of proposed hybrid precoding approaches the digital precoding in the term of total sum data rate as we increase number of RF chains and SNR.

\section{KEYWORDS}

Hybrid precoding, beamforming, MU-MIMO, Massive MIMO.

\section{INTRODUCTION}

Scarcity of spectrum has always been a problem in communication networks. Researchers always look adequate band for good propagation properties. Unfortunately, the current spectrum does not fulfill the need of bandwidth greedy broadband communications. For future wireless system such as $5 \mathrm{G}$, data rates for a user expected to increase explosively. Therefore, the researcher is working to move their attention to millimeter wave (mmWave) communications from microwave communications due to the availability of a huge amount of unused spectrum at mmWave. Communication at mmWave is targeting a speed in Gbits/s (data rates about $10 \mathrm{Gbits} / \mathrm{s}$, which is more than current $4 \mathrm{G} 100$ times) according to the IEEE 802.11 and WiGig. However, operating at mmWave comes with the high signal attenuation and scattering limitations. To overcome the issue of signal attenuation many researchers have proposed a new small cell system like micro-cell, femto-cell and pico-cell. By the small cells, we can combine mmWave and MIMO system to perform 3D beamforming easily. Directional communication which requires transmit and receive antenna beamforming can improve signal-to-noise ratio (SNR) to the sufficient level in the mmWave bands. Implementation of the massive MIMO at mmWave for beamforming enables us to install hundreds or thousands of antennas at the base station and receiver, which increases SNR at the receiver, consequently, a significant increase in system capacity can be observed. With digital beamforming, multiple streams can be transmitted in parallel, however it requires large numbers of radio frequency (RF) chains and analog to digital converters (ADCs) which makes the digital beamforming more complex and costly in the term of power consumptions. While analog precoding has less complexity and can be implemented with the phase shifter that controls the phases of transmit signal, but it supports only one data stream with high SNR, resultantly the capacity cannot be increased significantly. However, combining of both the schemes 
analog and digital precoding, enables us to get the advantages of the both. Therefore, hybrid beamforming becomes a hot research issue [1].

While designing precoding schemes for mmWave massive MIMO system, mostly researchers consider fully connected structure [2]. Many algorithms are used for different structures for hybrid precoding. Assuming perfect channel knowledge at the transmitter, a low-complexity iterative algorithm based on orthogonal matching persuit (OMP) is proposed in [3]. The hybrid technique provides a trade-off between the analog precoding that offers low-complexity with limited-performance and digital precoding having high-complexity with good-performance [4][5]. One of the most advantages of hybrid scheme is that it can deal with the less number of RF chains and more number of antennas [6]. In [7], the spares nature of the mmWave channel is studied. Following [6], the authors in [2] designed hybrid precoding algorithm based on alternative minimization and matrix decomposition. In [8] system model investigates both fully connected structure (each antenna is connected to all RF chains) and partially connected structure. Based on spatially sparse channel, a hybrid precoding is proposed in [9] that solves the problem by spares approximation approach for achievable rate as the nearoptimal solution. A codebook based algorithm is proposed in [10] that involve an iterative search in predefined codebook to get the matrix of optimal hybrid precoding.

In the existing research, the most works on hybrid beamforming are focusing on single user MIMO (SU-MIMO) systems and trying to reduce the RF chains without affecting the multi-data streams. It is found that, the number of RF chains are still more than data streams, which makes the hybrid beamforming hard to be implemented and costly in the term of the power consumption. In our work, we proposed a novel hybrid precoding where a group of antenna serves a group of users simultaneously as a result the required number of RF chain are reduced. We design hybrid precoding for multiuser massive MIMO (MU-massive MIMO) in mmWave system as follows:

(i) First we design analog and digital decoder for each user.

(ii) Use effective channel to calculate analog precoder, which is same for all the users.

(iii) Adopt block diagonalization (BD) for mitigate the interference and intrainterference between the users.

(iv) By some available algorithms, we design analog RF precoder system.

(v) Digital precoding designed by $\mathrm{BD}$ maximizes the total rate for multiuser massive MIMO system in downlink channel.

(vi) Hybrid beamforming has been design by weighted sum mean Square error (WSMSE) for which is the inverse gain of its digital precoding.

(vii) Finally, we study the performance of both digital and proposed hybrid beamforming with different parameters like SNR, data streams and RF chains.

The rest of the paper is organized as follows. Massive MIMO and mmWave communications are studied in detail in Section 2. System model and channel model are described in Section 3. The hybrid precoding design is explained in Section 4, while Section 5 narrates simulation results. Finally, the conclusions are made in Section 6. 


\section{MASSIVE MIMO AND MILLIMETER WAVE COMMUNICATIONS}

MIMO has become integral part of wireless standards as it can increase the reliability and throughput of the wireless communication systems [11] and one of the most important key for current $4 \mathrm{G}$ wireless systems. Initially, MIMO was designed for the single-user, therefore, SU-MIMO become the integral part of long-term evaluation and WiMAX [12]. Later it shifted from single-user to multi-user MIMO system [12] where base station can communicate with a group of users simultaneously with multiple antennas. To meet increasing data rates and traffic demand, currently, massive MIMO has become hot research issue where hundreds or thousands of antennas are installed at the base station to serve the group of users and minimize the interference. The mmWave band is emerged as most favorable band for massive MIMO.

\subsection{Benefits of MU- Massive MIMO}

MU-massive MIMO has attained a lot of attention for coming $5 \mathrm{G}$ wireless systems because of its high data rates for all the users and high reliability [13]. The multiple antennas that are installed at transmitter or receiver support multi signal paths to get large throughput. By adopting MU-massive MIMO technique the system capacity can be improved by factor of 10 and spectral efficiency by factor of 20 when the numbers of antennas installed at transmitter and receiver are about 256 [3]. Many more benefits can be achieved which are as follows:

i. Energy efficiency (EE) in modern cellular communications have growing attention worldwide. New research approach relative to EE called green cellular system has driven the mobile operators to meet the requirements of clean environment, decrease the cost and find solutions to improve the EE for cellular mobiles [14].

ii. In MU-Massive MIMO, we can reduce the numbers of amplifiers that uses a huge energy by replacing the traditional power amplifiers by a hundreds of low-cost power amplifiers [13], [15].

iii. Massive MIMO has a huge overflow of degree of freedom. Suppose we have 128 antennas that serve 28 users, then 100 degree of freedom remained unused [16].

\subsection{Estimation of Channel}

Channel state information (CSI) is important for improving the gain of multi-antenna in MIMO system. Therefore, CSI becomes more complicated for massive MIMO system because the huge numbers of antenna at base station. In addition, massive MIMO either applying FDD or TDD requires a large numbers of pilots. In the presence of large numbers of users, the reuse of same set of orthogonal pilots in the neighboring cells can result the pilot contamination in multi-cell system [17]. The pilot contamination limits the performance and decays the capacity of massive MIMO system. When the number of antennas are increased at base station, the SINR at receiver side will increase too. Because the number of antennas at base station are equal to the number of pilot contaminations, the data signals for all users decreased. To solve this problem, the SIC is applied to mitigate the interference between the pilot and data signals [18]. 


\subsection{Communications in Millimeter Waves}

Currently, all of the mobile system utilize spectrum in the range of $300 \mathrm{MHz}$ to $30 \mathrm{GHz}$. The advancement in electronic devices fabrication allows us to utilize the mmWave spectrum having large bandwidth for fifth generation (5G) wireless system networks. Therefore, the $5 \mathrm{G}$ wireless system must test the 30 to $300 \mathrm{GHz}$ spectrum for the broadband communication system. However, the issues and challenges in the use of mmWave spectrum need to be addressed [19]. Availability of huge bandwidth in mmWave and provision of a hundreds and thousands of antennas at base station make mmWave and massive MIMO promising candidates for (5G) [1] [20].

The high-path loss due to penetration and atmospheric attenuation become the nightmare for the mmWave. Many researches have investigated it and advised to adopt small cell system networks such as femtocell, microcell to overcome the high-path loss [21]. One of the most suitable solutions to avoid the high atmospheric attenuation transported by mmWave is to utilize huge numbers of antennas to the base station. In past and current wireless systems omnidirectional antenna array are used. The deployment of massive set of narrow-beam directional antennas can steer the signal direct to the multiple users. There are a lot of antenna array techniques but the most two fundamental techniques are the beamforming and spatial multiplexing. In spatial multiplexing, multiple separately and independent encoded data streams can be transmitted by using multiple antenna elements and the receivers have ability to reconstructing the original signal effectively [13, 22-25]. Spatial multiplexing is more effective in two cases:

i. When the path of prorogation is rich, it can support multiple data streams transmission.

ii. At high channel SNR the strength of the original signal is not be affected when it splits in to multiple data streams.

In beamforming approach, the base station adapts the weight of for each array of antenna elements to control the data streams direction [13]. In this way, the data stream targets the desired user and not interfere the others. Beamforming is suitable in the conditions bellow:

i. When the SNR of the channel is low.

ii. When the power of the channel is limited.

iii. Beamforming is critical for mmWave to avoid the high-path loss due to the small wavelength.

\section{SYSTEM MODEL}

We consider downlink multiuser channel for hybrid and digital precoding, where one base station (BS) serves $M$ users in the cell. BS has $N_{t}$ transmit antennas and each user equipped with $N_{r}$ receive antennas. Number of streams $\ell$ for each user are limited by $\ell \leq N_{r}$. Total number of RF chains $N_{r f}$ at transmitter and total number of streams $M \times \ell$ in the downlink are limited by $\min \left(N_{t}, M \times N_{r}\right)$ and $\min \left(N_{r f}, M \times N_{r}\right)$ respectively. The $\mathrm{RF}$ chains at receiver are assumed equal to receiver antennas. The signal received at the $m^{\text {th }}$ user is given by: 


$$
y=H_{m}^{M} W_{m} F_{m} \sqrt{P_{m} d_{m}}+\sum_{j \neq m}^{M} H_{m}^{M} W_{j} F_{j} \sqrt{P_{j} d_{j}}+n_{m}
$$

Where $n_{m}$ is vector of i.i.d. $C N\left(0, \sigma^{2}\right)$ noise and $P_{m}$ power allocated to the $m^{\text {th }}$ user.

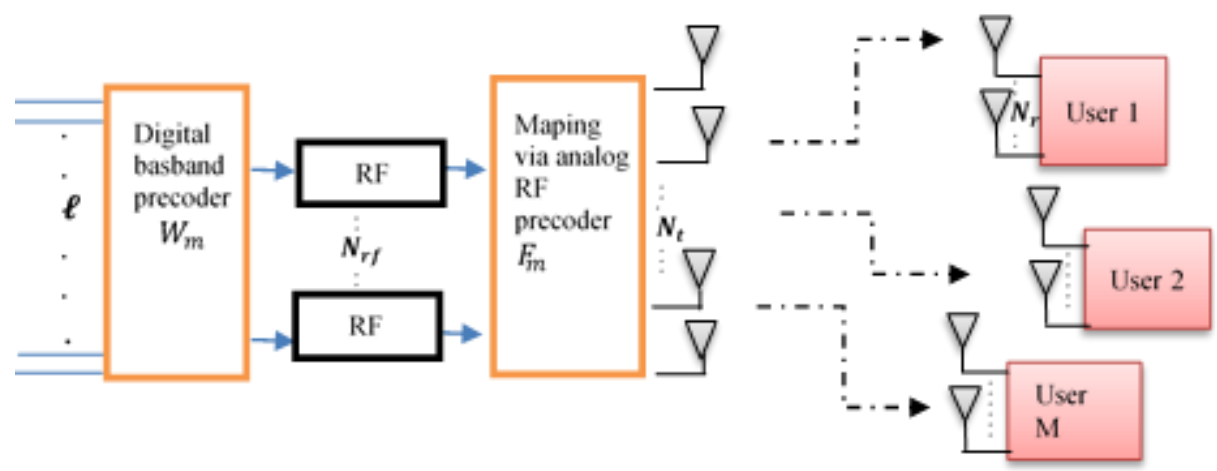

Figure 1. Proposed MU-Massive MIMO System.

\subsection{System Model for Digital Downlink Massive MIMO}

In digital downlink massive MIMO system, the BS uses precoding matrix $W=\left[W_{1}^{D}, W_{2}^{D}, \ldots \ldots ., W_{M}^{D}\right]$ at the transmitter where $W_{m}^{D} \in \square^{N_{t} \times \ell}$ is the matrix for $m^{t h}$ user. A linear matrix $G_{m}^{D} \in \square^{N_{r} \times \ell}$ may be used at the $m^{\text {th }}$ user to recover its symbols $d_{m}$ as:

$$
d_{m}^{D}=G_{m}^{D}\left(H_{m}^{H} W_{m} d+n_{m}\right)=G_{m}^{D}\left(H_{m}^{H} \sum_{i}^{M} W_{i} d_{i}+n_{m}\right)
$$

Where $H_{m}^{H} \in \square^{N_{t} \times N_{r}}$ is the massive MIMO channel between the $m^{\text {th }}$ receiver.

\subsection{System Model for Hybrid Downlink Massive MIMO}

Hybrid beamforming combines analog and digital precoders and decoders. If we expand the beamforming for multiuser massive MIMO, the estimated signal at $m^{\text {th }}$ user can written as:

$d_{m}^{H y}=G_{m}^{H y} R_{m}\left(H_{m}^{H} \sum_{i}^{M} F_{i} W_{i}^{H y} d_{i}+n_{m}\right)$

Where $W_{m}^{H y} \in \square^{N_{r f} \times \ell}, F_{m} \in \square^{N_{t} \times N_{r f}} \quad R_{m} \in \square^{N_{r} \times N_{r}}$ and $G_{m}^{H y} \in \square^{N_{r} \times \ell}$ are the matrices of baseband and analog precoding, and analog and digital decoding respectively. Every element of $F_{m} / R_{m}$ got a constant modulus. For simplicity, we denote $W_{m}^{H y}$ and $G_{m}^{H y}$ as $W_{m}$ and $G_{m}$ for further derivations. If we denote $A_{m}=G_{m} R_{m} H_{m}^{H} F_{m} W_{m}$ (Combining precoding and decoding of both digital and analog), then the received SNR at $m^{\text {th }}$ user is:

$$
\gamma_{m, t}=\frac{\left\|A_{m}(t, t)\right\|^{2} P_{m}}{\sum_{i \neq u}^{\ell}\left\|A_{m}(t, t)\right\|^{2} Q_{m}+\sum_{j \neq m}^{M}\left\|A_{m} A^{j}(t, t)\right\|^{2} P_{j}+\sigma^{2}}
$$




\subsection{Channel Model}

There are high free-space path losses in mmWave propagation environment that must be characterized by an appropriate channel model. We adopt cluster channel, described in [11]. This model performs the mmWave channel matrix as:

$$
H=\sqrt{\frac{N_{t} N_{r}}{N_{c l} N_{r a y}}} \sum_{i=1}^{N_{c l}} \sum_{l=1}^{N_{r a y}} \alpha_{i l} a_{r}\left(\phi_{i l}^{r} \theta_{i l}^{r}\right) a_{t}\left(\phi_{i l}^{t} \theta_{i l}^{t}\right)^{H}
$$

Where $N_{c l}$ is the numbers of clusters, $N_{\text {ray }}$ the numbers of rays for each cluster and the gain of $l^{\text {th }}$ ray in the $i^{\text {th }}$ prorogation channel is representing by $\alpha_{i l}$.

\section{PROBLEM FORMULATION AND HYBRID PRECODING DESIGN}

We want maximize the total sumrate of the system, which is given as:

$$
\begin{aligned}
& \arg _{W_{m} F_{m} R_{m} G_{m}} \max \sum_{m}^{M} \sum_{t=u}^{\ell} \log \left|1=\gamma_{m, t}\right| \\
& \text { s.t. } \sum_{m}^{M}\left\|W_{m} F_{m}\right\| \leq M \ell
\end{aligned}
$$

The above problem is non-convex and it is difficult to obtain its optimal solution. Therefore, we propose to solve an approximation of (6) in order to find near-optimal solution that can be practically implemented. First, we fix analog precoder and define effective channel matrix

$$
H_{\text {eff }}=H_{m}^{M} F_{m}
$$

We take SVD of effective channel for digital precoder and decoder design.

$$
H_{\text {eff }}=U_{\text {eff }} A_{\text {eff }} V_{\text {eff }}^{*}
$$

$$
W_{m}=V_{\text {eff }} \quad \text { and } F_{m}=U_{e f f}^{*}
$$

We design the hybrid precoding in two steps, starting with analog and digital decoders and then analog and digital precoder by the help of orthogonal algorithm. As we can note from equation (3) the matrices of hybrid are more constrained than the matrices of digital beamforming, which means digital beamforming achieves the best performance.

Due to the advantages of block diagonalization, we employ the $d_{m}^{D}$ as the received reference signal. We set the characteristics of proposed hybrid beamforming by finding the Euclidian distance between the received signal for digital and hybrid beamforming. It can be acquired by estimating the mean square error between the $d_{m}^{D}$ and $d_{m}^{H y}$ that is given by [1]:

$$
\xi_{m}=\mathrm{E}\left[\left(d_{m}^{D}-d_{m}^{H y}\right)\left(d_{m}^{D}-d_{m}^{H y}\right)^{H}\right]
$$

We use the block diagonalization digital beamforming for total sum rate maximization of multiuser massive of downlink channel. The block diagonalization uses three basic steps as follows:

i- Firstly, it eliminates the interference of other users

ii- Secondly it cancels the self-interference for each desired user. 
iii- It optimizes the power of each symbol for maximizing the total sum rate. For better clarification of the above steps we can define these as follows:

$$
\begin{aligned}
& \hat{H}_{m} \square\left[H_{1}, H_{2}, \ldots \ldots H_{m-1}, H_{m+1}, \ldots H_{M}\right] \\
& V_{h 0 m} \square \operatorname{null}\left(\hat{H}_{m}^{H}\right) \\
& X_{m} \square H_{m}^{H} V_{h 0 m}=\hat{U}_{m h} Z_{m} \hat{V}_{h m}^{H}
\end{aligned}
$$

Where $V_{h 0 m} \in \square^{N_{t} \times \ell}$ is the first factor of $\ell$ of $\operatorname{null}\left(\hat{H}_{m}^{H}\right), \quad \hat{U}_{m h} \in \square^{N_{r} \times \ell}, \hat{V}_{h m}^{H} \in \square^{\ell \times \ell}$ are unitary matrices and $Z_{m}$ is the diagonal matrix of size $\ell$. As we mention above the BD beamforming algorithm can be carry out by putting $W_{m}$ and $G_{m}$ of (2) as $W_{m}=V_{h 0 m} \hat{V}_{\mathrm{h} 0 m}^{H} \sqrt{Q_{m}}$ and $G_{m}=\hat{U}_{h m}$, the $Q_{m}$ is denoted the matrix of power allocation for each user. Therefore, the relation between the input-output in (2), can be rewritten as:

$\hat{d}_{m}^{D}=Z_{m} \sqrt{Q_{m}} d_{m}+\hat{U}_{m h}^{H} n_{m} \Rightarrow \hat{d}_{m i}^{D}=z_{m i} \sqrt{q}_{m i} d_{m}+\hat{u}_{m h i}^{H} n_{m}$

Where the $\hat{d}_{m i}^{D}$ denoted the elements of ${ }^{\hat{d}_{m}^{D}}$ and the diagonal element is represented by $z_{m i}\left(q_{m i}\right)$ of $Z_{m} Q_{m}$ and finally, the $\hat{u}_{m h i}^{H}$ denoted the row of $\hat{U}_{m h}^{H}$.

The final steps of $\mathrm{BD}$ can be performed by solving the total sum rate maximization as:

$\max \left(q_{m i}\right)=\sum_{m=1}^{M} \sum_{i=1}^{\ell} \log _{2}\left(1+\frac{z_{m i}^{2} q_{m i}}{\sigma^{2}}\right)$

s.t. $\sum_{m=1}^{M} \sum_{i=1}^{\ell} q_{m i}=P_{\max }$

The elements of maximum power at transmitter $P_{\max }$ is represented by the $q_{m i}$. The aim is to solve this problem by utilize some simple algorithms such as water filling algorithm to reach the close-optimal solution.

Form the above equations we can recognize that, the BD beamforming $R_{m m}=Z_{m} \sqrt{Q_{m}}$ and $R_{m i}=0, \forall m \neq i$. Therefore, the $\xi_{m}$ in (10) can be written as:

$$
\begin{aligned}
& \xi_{m}=\left(\hat{G}_{m}^{H} R_{m}^{H} H_{m}^{H} F_{m} \hat{W}_{m}-Z_{m} \sqrt{Q_{m}}\right)-\left(\hat{G}_{m}^{H} R_{m}^{H} H_{m}^{H} F_{m} \hat{W}_{m}-Z_{m} \sqrt{Q_{m}}\right)^{H}+ \\
& \sum_{i=1, i \neq m}^{M}\left(\hat{G}_{m}^{H} R_{m}^{H} H_{m}^{H} F_{m} \hat{W}_{i}\right)\left(\hat{G}_{m}^{H} R_{m}^{H} H_{m}^{H} F_{m} \hat{W}_{m}\right)^{H}+\sigma^{2}\left(\hat{G}_{m}^{H} R_{m}^{H}-\hat{U}_{h m}\right)\left(\hat{G}_{m}^{H} R_{m}^{H}-\hat{U}_{h m}\right)^{H}
\end{aligned}
$$

Now we have to design analog precoder $F_{m}$, digital precoder $\hat{W}_{m}$ and analog decoder $R_{m}$ . The gap between digital and hybrid beamforming is constant for all the symbols. We use WSMSE algorithm to optimize the main objective function i.e. the $i^{i^{t h}}$ symbol weight of the $m^{\text {th }}$ user is set to $\frac{1}{z_{m i}^{2} q_{m i}}$, and the equation can be mathematically solved as:

$$
\min _{F_{m} \hat{W}_{m} R_{m}} \sum_{m=1}^{M} \operatorname{tr}\left\{\left(z_{m} \sqrt{Q_{m}}\right)\right\}^{-1} \xi_{m}\left\{\left(z_{m} \sqrt{Q_{m}}\right)\right\} \square \xi_{m}
$$


s.t. $\quad \sum_{m=1}^{M} \operatorname{tr}\left\{F_{m} \hat{W}_{m} \hat{W}_{m}^{H} F_{m}^{H}\right\}=Q_{\max }$

$$
\left|F_{m(i, j)}\right|^{2}=M, \quad\left|R_{m(i, j)}\right|^{2}=M
$$

The $\sum_{m=1}^{M} \operatorname{tr}\left\{F_{m} \hat{W}_{m} \hat{W}_{m}^{H} F_{m}^{H}\right\}=Q_{\max }$ is introduced to make the same total power for hybrid and digital beamforming. We further optimize the problem in two steps:

i. First optimize the receiving matrix (digital decoder) and analog decoder for each user.

ii. Then optimized analog and digital precoders for fixed analog and digital decoders.

\subsection{First Step}

In the first step, we optimized jointly analog $R_{m}$ and digital $G_{m}$ decoders for $m^{\text {th }}$ user, we written it as:

$\min _{\overline{\bar{W}}_{m} F_{m}}\left|\overline{\bar{W}}_{m} F_{m}-\overline{\bar{U}}_{h m}\right|_{F}^{2}$, s.t. $\left|F_{m}\right|^{2}=1$

As we observed that $\overline{\bar{W}}_{m}$ and $F_{m}$ are joint function. Therefore, the constraint of the objective function is non-convex and optimal solution of objective function is difficult to find. To solve function in (17) we set the variable of $\bar{F}_{m}=\mathbf{f}_{m}$ where the $\mathbf{f}_{m}=\left[F_{m} n u l l\left(\bar{F}_{m}^{T}\right)\right]$ and rewrite the (17) as:

$$
\min _{G_{m}}\left|\bar{R}_{m} \bar{G}_{m}-\overline{\bar{U}}_{h m}\right|_{F}^{2} \text {, s.t. }\left\|\operatorname{diag}\left(\bar{R}_{m} \bar{R}_{m}^{H}\right)\right\|_{0}=N_{r f}
$$

But even after some mathematical manipulation the non-convex problem is still the same. The better solution to overcome non-convex problem is to use the orthogonal matching pursuit algorithm as given below: 


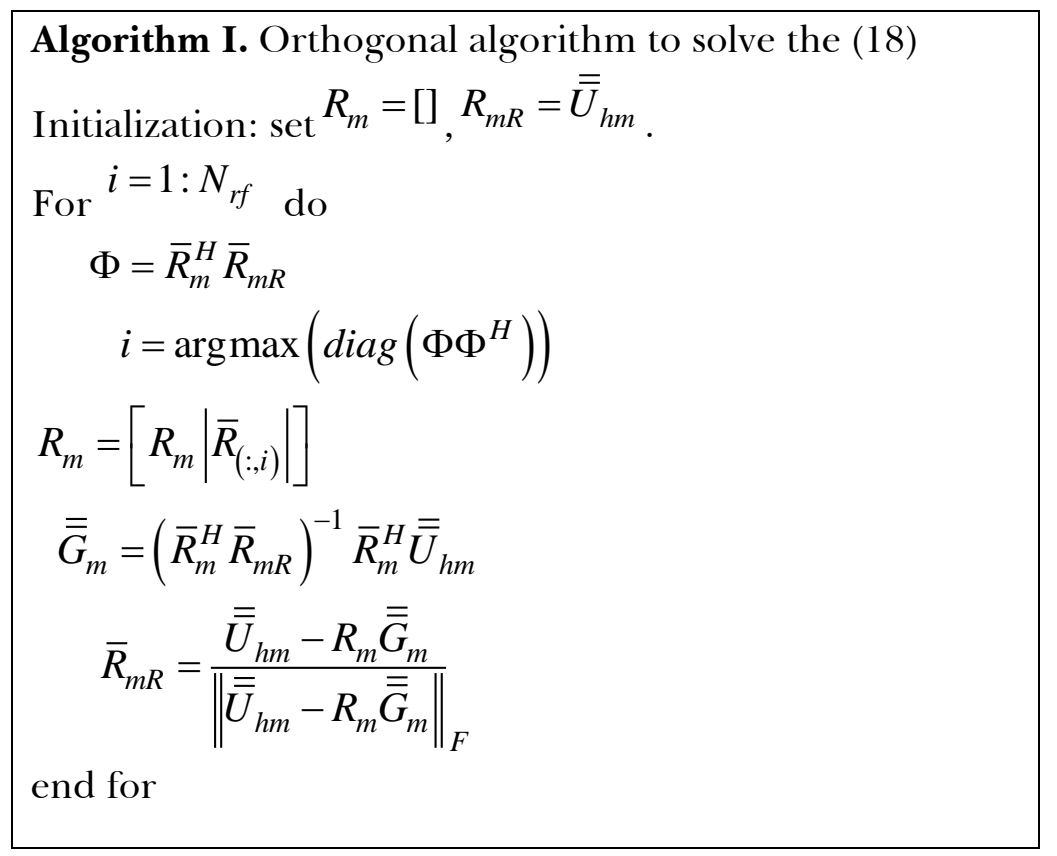

\subsection{Second Step}

For a given $\overline{\bar{G}}_{m}$ and $\overline{\bar{R}}_{m}$ in (18), the problem can be reformulated as:

$$
\begin{gathered}
\max _{F_{m} \hat{W}_{m}}\left\|\overline{\bar{G}}_{m} R_{m}^{H} H_{m}^{H} F_{m} \bar{W}_{m}-I\right\|_{F}^{2}, \\
\text { s.t. } \operatorname{tr}\left\{F_{m} W_{m} W_{m}^{H}\right\} \leq P_{\max } \\
\left|F_{(i, j)}\right|^{2}=1
\end{gathered}
$$

We can notice form (15) that the correlation of analog precoder is much high with right singular value of ${ }_{m}^{H}$. It is assumed that each column of analog precoder ${ }^{F_{m}} \mathbf{F}_{\mathbf{m}}$ is derived from one of the column of $\mathbf{f}_{m}$, where $\mathbf{f}_{m}=\left[F_{m} \operatorname{null}\left(\bar{F}_{m}^{T}\right)\right] \in \square^{N_{t} \times N_{t}}$ with normalized entries. Hence the modulus value of analog precoder $\mathbf{f}_{m}$ is set to 1 . By setting $\bar{F}_{m}=\mathbf{f}_{m}$ and applying the same technique as step1, the problem (19) can be rearrange as

$$
\max _{\bar{F}_{m}}\left\|\left(R_{m} H_{m} \overline{\bar{G}}_{m}\right)^{H} \bar{F}_{m} \bar{W}_{m}-I\right\|_{F}^{2}
$$

s.t. $\operatorname{tr}\left\{\bar{F}_{m} \bar{W}_{m} \bar{W}_{m}^{H} F_{m}^{H}\right\}=P_{\max }$

$\left\|\operatorname{diag} \bar{W}_{m} \bar{W}_{m}^{H}\right\|=N_{r f}$

Therefore, the solution to this problem can be found using the orthogonal algorithm as used for (18) in step 1, as follows: 


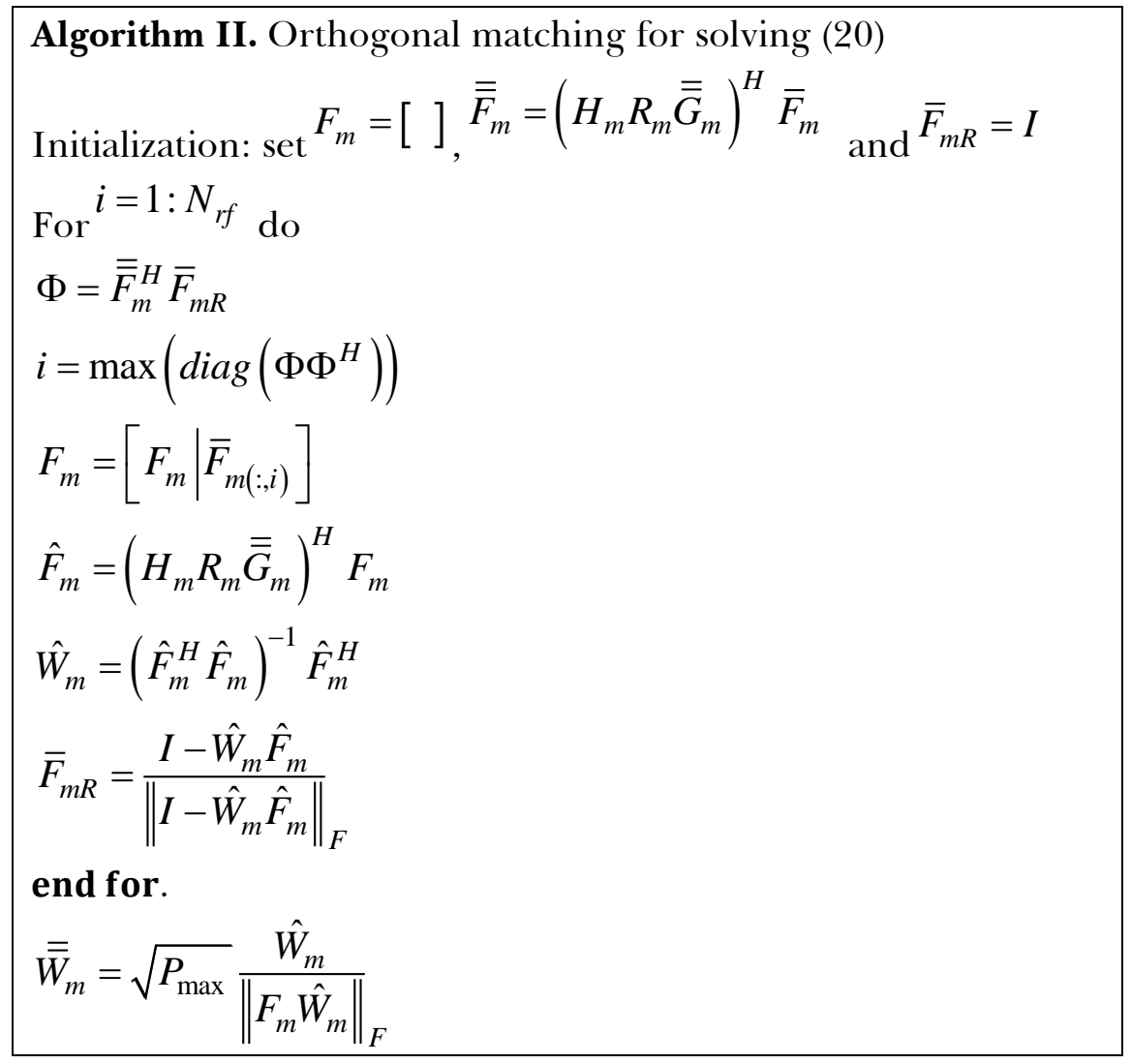

It is mentioned that the step 1 and step 2 both use fix the power at transmitter side.

\section{SIMULATION RESULTS}

MIMO This section presents the simulation results. We use, $N_{t}=128, N_{r}=32$, where is $N_{t}$ is the number of antenna at BS and $N_{r}$ is the number of antennas at each user's equipment. The number of users are $M=4$, antenna spacing $d=0.5 \lambda$, the number of paths are $L=16$. The $S N R=\frac{P_{a v}}{\sigma^{2}}$ is dominated by $\sigma^{2}$ where $P_{a v}=\frac{P_{\max }}{\ell}$ and the total power is limited by $P_{\max }=M \ell$. The channel parameters

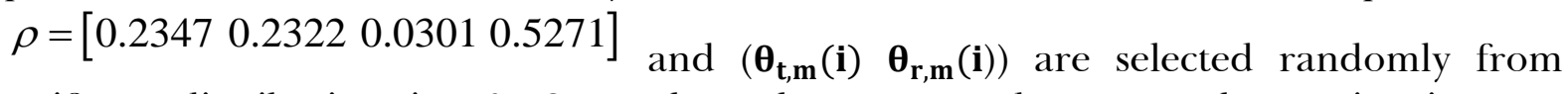
uniform distribution in $[0,2 \pi]$. Then the computed sum total rate is given as $R_{t}=\sum_{m=1}^{M} \sum_{i=1}^{\ell} R_{m i}$, which means the sum total rate for each symbol is computed by $R_{m i}=\log _{10}\left(1+\gamma_{m i}\right)$, where $R_{m i}$ is the achieved SINR of $m^{\text {th }}$ user $i^{\text {th }}$ stream. Each plot is generated by 1000 iterations, $\forall M$.

\subsection{Compression between Hybrid and Digital Beamforming}

In this part, we compare the performance of hybrid beamforming with the digital beamforming. For simulation, we set the data streams $\ell$ to 12 and the number of radio frequency chains $N_{r f}$ to 16 . The achievable rates for the digital and hybrid beamforming are shown in Figure 2 It can be observed that hybrid beamforming performance 
approaches the digital beamformig performance from low to medium SNR region. In addition, it can be seen the small gap performance may occur at high SNR region.

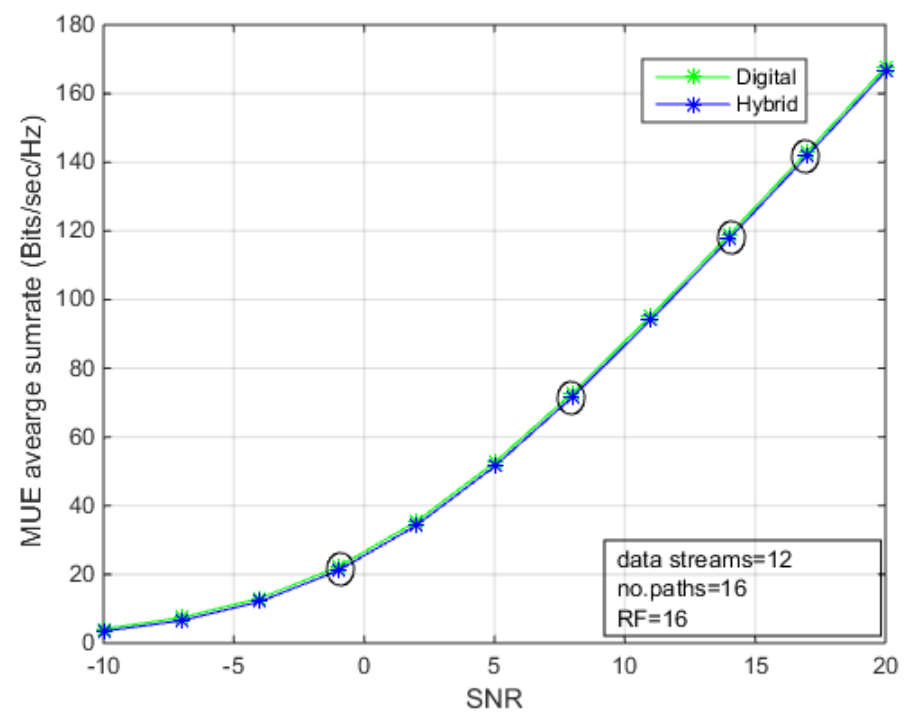

Figure 2. Digital and Hybrid Beamforming Compression.

\subsection{J oint Effects of $\ell$ and $N_{r f}$ On Hybrid and Digital Beamforming}

In this section we study the joint effect of data streams and radio frequency chains $N_{r f}$ on both hybrid and digital beamforming performance. We set $\mathrm{SNR}=5 \mathrm{~dB}$. Figure 3 shows the performance comparison of both schemes. It can be noted that by increasing the data streams $\ell$, the achievable sum rate of hybrid and digital beamforming will also increase. The data streams are limited by the constant of maximum power $P_{\max }=M \ell$. By decreasing the data streams, the performance gap between digital and hybrid can be bridged. If we limit the number of radio frequency chain $N_{r f}$ and the analog to digital converters (ADCs), the performance gab between both schemes digital and hybrid will decrease by reducing the number of streams $\ell$.

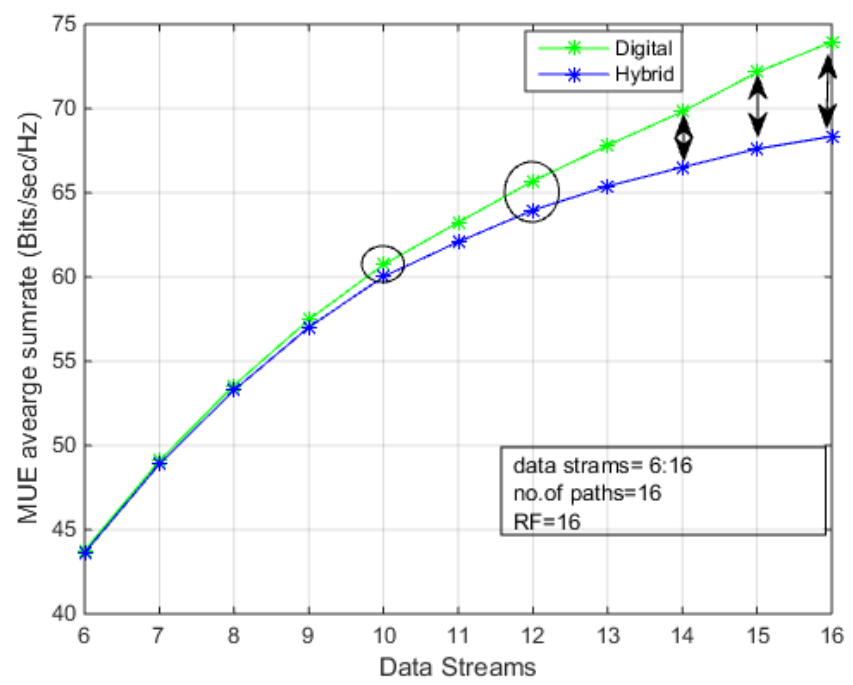

Figure 3. Joint Effects Of $\ell$ and $N_{r f}$ On Digital and Hybrid Beamforming. 


\subsection{Effect of ${ }^{N_{r f}}$ on hybrid beamforming}

In this part, the performance of the hybrid beamforming is examined by varying the number of RF chains. Figure 4 presents of hybrid algorithm for deferent setting of $N_{r f}=8: 20$, , at $\mathrm{SNR}=5 \mathrm{~dB}$ and $\ell=8$. We can see that by increasing the number of RF chains and ADCs, the performance of the hybrid algorithm can be improved significantly and the gap between the two schemes becomes small and almost constant when the number of RF chains are more than the twice of the data streams.

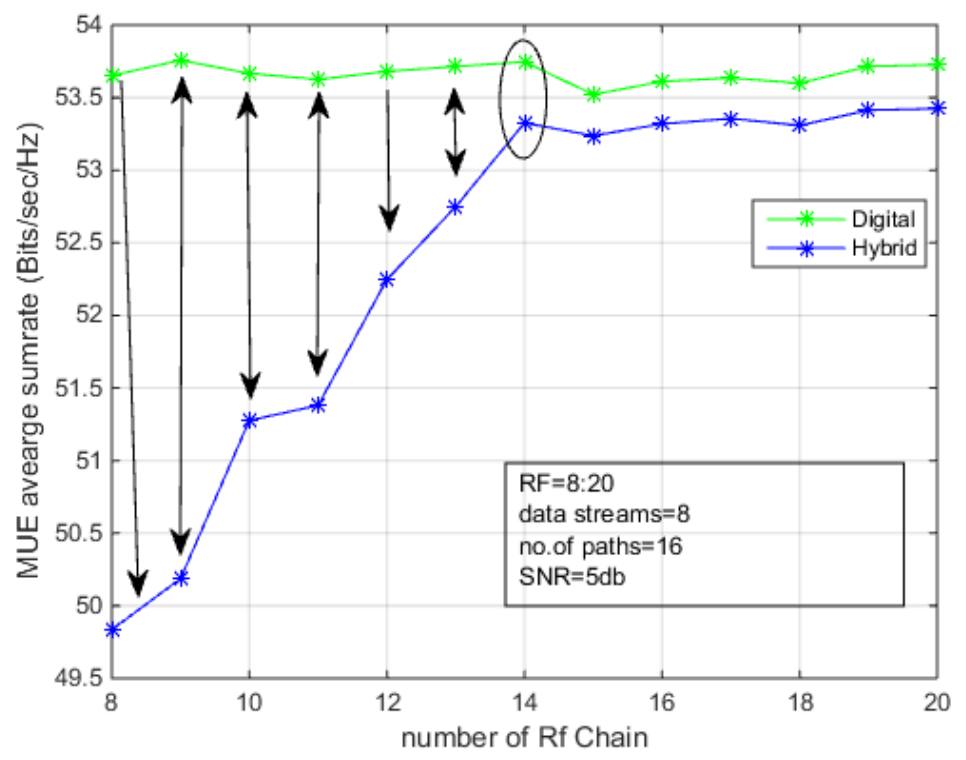

Figure 4. Effect of $N_{r f}$ on the Hybrid Beamforming.

\section{CONCLUSION}

We have designed a hybrid beamforming scheme for downlink MU-massive MIMO system. Hybrid scheme is indirectly designed by using WSMSE to maximize the total sumrate, whereas conventional block diagonalization is used for digital beamforming. We examine the relation between two by considering different parameters such as SNR, number of data symbols and the number of RF chains and ADCs. From the result we conclude that the performance gap between hybrid and digital beamforming can be bridged by decreasing the number of data streams for a given number of ADCs and RF chains. On the other hand, when the number of data streams are fixed, an increase in $\mathrm{RF}$ chains and $\mathrm{ADC}$ increases the total sum rate of hybrid scheme that we expect. The hybrid beamforming reaches the same performance of digital beamforming when SNR reaches from the low to the high region. 


\section{REFERENCES}

[1] T. E. Bogale and L. B. Le, "Beamforming for Multiuser Massive MIMO Systems: Digital Versus Hybrid Analog-Digital," Proceedings of the IEEE Global Communications Conference, Austin, USA, (2014) December 8-12.

[2] Z. Xinying, A. F. Molisch, and K. Sun-Yuan, "Variable-Phase-Shift-Based RFBaseband Codesign for MIMO Antenna Selection," IEEE Transactions on Signal Processing., vol. 53, no. 11, (2005), pp. 4091-4103.

[3] Y. Gao, M. Khaliel, and T. Kaiser, "Wideband Hybrid Analog-Digital Beamforming Massive MIMO Systems Based on Rotman Lens," Proceedings of the IEEE International Conference on Communication Systems (ICCS), Shenzhen, China, (2016) December 14-16.

[4] O. El Ayach, R. W. Heath, S. Abu-Surra, S. Rajagopal, and Z. Pi, " Low Complexity Precoding for Large Millimeter Wave MIMO Systems," Proceedings of the IEEE International Conference on Communications (ICC), Ottawa, Canada, (2012) June $10-15$.

[5] E. Zhang and C. Huang, "On Achieving Optimal Rate of Digital Precoder by RFBaseband Codesign for MIMO Systems," Proceedings of the IEEE 80th Vehicular Technology Conference (VTC2014-Fall), Vancouver, Canada, (2014) September 14-17.

[6] S. He, C. Qi, Y. Wu, and Y. Huang, "Energy-Efficient Transceiver Design for Hybrid Sub-Array Architecture MIMO Systems," IEEE Access., vol. 4, pp. 98959905.

[7] A. Li and C. Masouros, "Hybrid Analog-Digital Millimeter-Wave MU-MIMO Transmission with Virtual Path Selection," IEEE Communications Letters., vol. 21, no. 2, (2017), pp. 438-441.

[8] S. Park, A. Alkhateeb, and R. W. Heath, "Dynamic Subarrays for Hybrid Precoding in Wideband mmWave MIMO Systems," IEEE Transactions on Wireless Communications., vol. 16, no. 5, (2017), pp. 2907-2920.

[9] R. Rajashekar and L. Hanzo, "Hybrid Beamforming in mm-Wave MIMO Systems Having a Finite Input Alphabet," IEEE Transactions on Communications., vol. 64, no. 8, (2016), pp. 3337-3349.

[10] D. Vouyioukas, "A Survey on Beamforming Techniques for Wireless MIMO Relay Networks," International Journal of Antennas and Propagation., (2013), pp.1- 21.

[11] Z. Pi and F. Khan, "An Introduction to Millimeter-Wave Mobile Broadband Systems," IEEE Communications Magazine., vol. 49, no. 6, (2011), pp. 101-107.

[12] M. K. Samimi and T. S. Rappaport, "3-D Statistical Channel Model for MillimeterWave Outdoor Mobile Broadband Communications," Proceedings of the IEEE International Conference on Communications (ICC), London, UK, (2015) June 812.

[13] J. Wu, Y. Zhang, M. Zukerman, and E. K. N. Yung, "Energy-Efficient Base-Stations Sleep-Mode Techniques in Green Cellular Networks: A Survey," IEEE Communications Surveys \& Tutorials., vol. 17, no.2, (2015), pp. 803-826. 
[14] S. Han, C. 1. I, Z. Xu, and C. Rowell, "Large-Scale Antenna Systems With Hybrid Analog and Digital Beamforming for Millimeter Wave 5G," IEEE Communications Magazine, vol. 53, no.1, (2015), pp. 186-194.

[15] L. Dai, X. Gao, J. Quan, S. Han, and I. C. L, "Near-Optimal Hybrid Analog and Digital Precoding for Downlink mmWave Massive MIMO Systems," Proceedings of the IEEE International Conference on Communications (ICC), London, UK, (2015) June 8-12.

[16] A. Osseiran, F. Boccardi, V. Braun, K. Kusume, P. Marsch, M. Maternia. O. Queseth, M. Schellmann, H. Schotten, H. Taoka and H. Tullberg, "Scenarios for 5G Mobile and Wireless Communications: The Vision of the METIS Project," IEEE Communications Magazine., vol. 52, no. 5, (2014), pp. 26-35, 2014.

[17] X. Yu, J. C. Shen, J. Zhang, and K. B. Letaief, "Alternating Minimization Algorithms for Hybrid Precoding in Millimeter Wave MIMO Systems," IEEE Journal of Selected Topics in Signal Processing., vol. 10, no, 3, (2016), pp. 485-500.

[18] A. Alkhateeb, O. E. Ayach, G. Leus, and R. W. Heath, "Channel Estimation and Hybrid Precoding for Millimeter Wave Cellular Systems," IEEE Journal of Selected Topics in Signal Processing., vol. 8, no, 5, (2014), pp. 831-846.

[19] O. E. Ayach, S. Rajagopal, S. Abu-Surra, Z. Pi, and R. W. Heath, "Spatially Sparse Precoding in Millimeter Wave MIMO Systems," IEEE Transactions on Wireless Communications., vol. 13, no, 3, (2014), pp. 1499-1513.

[20] M. N. Khan and M. Jamil, "Performance Improvement in Lifetime and Throughput of LEACH Protocol," Indian Journal of Science and Technology, vol. 9, no. 21, (2016), pp. 1-6.

[21] M. N. Khan and Jamil, "Maximizing Throughput of Free Space Communication Systems Using Puncturing Technique," Arabian Journal for Science and Engineering, vol. 39, no. 12, (2014), pp. 8925-8933.

[22] M. N. Khan, H. K. Hasnain and M. Jamil, "Digital Signal Processing: A Breadthfirst Approach”, Rivers Publishers, Denmark, (2016), pp. 221-247.

[23] J. G. Andrews, S. Buzzi, W. Choi, S. V. Hanly, A. Lozano, A. C. K. Soong, et al., "What Will 5G Be?," IEEE Journal on Selected Areas in Communications., vol. 32, no, 6, (2014), pp. 1065-1082.

[24] C. Li, Y. Ying, C. Xiaohui, and W. Weidong, "Multi-Stage Beamforming Codebook for $60 \mathrm{GHz}$ WPAN," Proceedings of the 6th International ICST Conference on Communications and Networking in China (CHINACOM), Harbin, China, (2011) August 17-19.

[25] M. H. Alsharif and R. Nordin, "Evolution towards Fifth Generation (5G) Wireless Networks: Current Trends and Challenges in the Deployment of Millimetre Wave, Massive MIMO, and Small Cells," Telecommunication Systems., vol. 64, no. 4, (2017), pp. 617-637. 
